\title{
Transitioning to a New Normal in the Post-COVID Era
}

\section{Di M. Jiang ${ }^{1} \cdot$ Alejandro Berlin ${ }^{2} \cdot$ Lesley Moody $^{3} \cdot$ Rajat Kumar $^{1} \cdot$ Breffni Hannon $^{4} \cdot$ Monika K. Krzyzanowska $^{1}$ • Neesha Dhani ${ }^{1} \cdot$ Heather Cole $^{5} \cdot$ Mary Elliott $^{6} \cdot$ Srikala S. Sridhar $^{1}$}

Published online: 22 June 2020

(C) Springer Science+Business Media, LLC, part of Springer Nature 2020

Keywords Pandemic $\cdot$ Coronavirus $\cdot$ Telemedicine $\cdot$ Cancer care facilities $\cdot$ Clinical trial $\cdot$ Mental health

The COVID-19 pandemic has placed significant strains on health care systems worldwide, especially on routine cancer care. Cancer patients, owing to their disease and treatment, are at increased risk for contracting COVID-19 and developing severe outcomes [1]. They are also at risk for cancer progression if left untreated, making their care increasingly complex during the pandemic [2].

The Princess Margaret Cancer Center (PM) in Toronto is the largest cancer center in Canada, providing specialized cancer care to a broad and diverse Canadian population. Building on prior experience from managing the biggest SARS (severe acute respiratory syndrome) outbreak outside of Asia in 2003 [3], a multidisciplinary steering committee was assembled to develop a pandemic strategy guided by principles of maintaining health, safety, and well-being of patients and staff, minimizing patient risk from both oncologic and infectious disease, and allocating resources in a just and equitable fashion based on need. The PM strategy had two main priorities: to mitigate exposure and limit spread and to create additional surge capacity to care for cancer patients affected by COVID-19.

Standfirst Various strategies have been implemented by cancer care institutions across the world in response to the COVID-19 pandemic. Looking ahead, many of these changes including virtual care, community-based care delivery models, and pragmatic trials will likely transform the way cancer care is delivered going forward.

Srikala S. Sridhar

srikala.sridhar@uhn.ca

1 Medical Oncology and Hematology, Princess Margaret Cancer Centre, University Health Network, University of Toronto, 610 University Avenue, Toronto, Ontario M5G 6M9, Canada

2 Radiation Oncology, Princess Margaret Cancer Centre, University Health Network, University of Toronto, Toronto, Ontario, Canada

3 Princess Margaret Cancer Centre, University Health Network, Toronto, Ontario, Canada

\section{Current Impacts on Cancer Care}

In line with institutional and Cancer Care Ontario guidelines [4], patients of PM are now triaged based on need. For each patient visit, an assessment is made whether it can be safely conducted virtually (telephone or videoconference through the Ontario Telemedicine Network). Many aspects of cancer care are also being shifted to the community, including essential laboratory, imaging evaluations, and home care services to mitigate exposure risk. In February 2020, less than 2\% of medical oncology ambulatory clinic activities were conducted virtually. In response to COVID-19, PM implemented virtual care capabilities across divisions in mid-March, as well as a novel, integrated digital tool to facilitate digital order entry and widespread adoption of virtual care on March 24. Overall, the goal was to shift at least $50 \%$ of in-person visits to virtual care, and this target was achieved within 4 days of launch, and increasing up to $71 \%$. The Division of Medical Oncology and Hematology quickly adopted virtual visits for the majority of patients (Fig. 1a). This, along with increased coordination of hospital appointments, has enabled in-

4 Supportive Care, Princess Margaret Cancer Centre, University Health Network, Toronto, Ontario, Canada

5 Cancer Clinical Research Unit, Princess Margaret Cancer Centre, University Health Network, Toronto, Ontario, Canada

6 Department of Psychiatry, Princess Margaret Cancer Centre, University Health Network, University of Toronto, Toronto, Ontario, Canada 
Fig. 1 a Increased utilization of virtual care in the Division of Medical Oncology and Hematology, Princess Margaret Cancer Center, from February 3 to May 24, 2020. OTN, Ontario Telemedicine Network. b Chemotherapy day care visits at the Princess Margaret Cancer Center, from February 3 to May 24, 2020
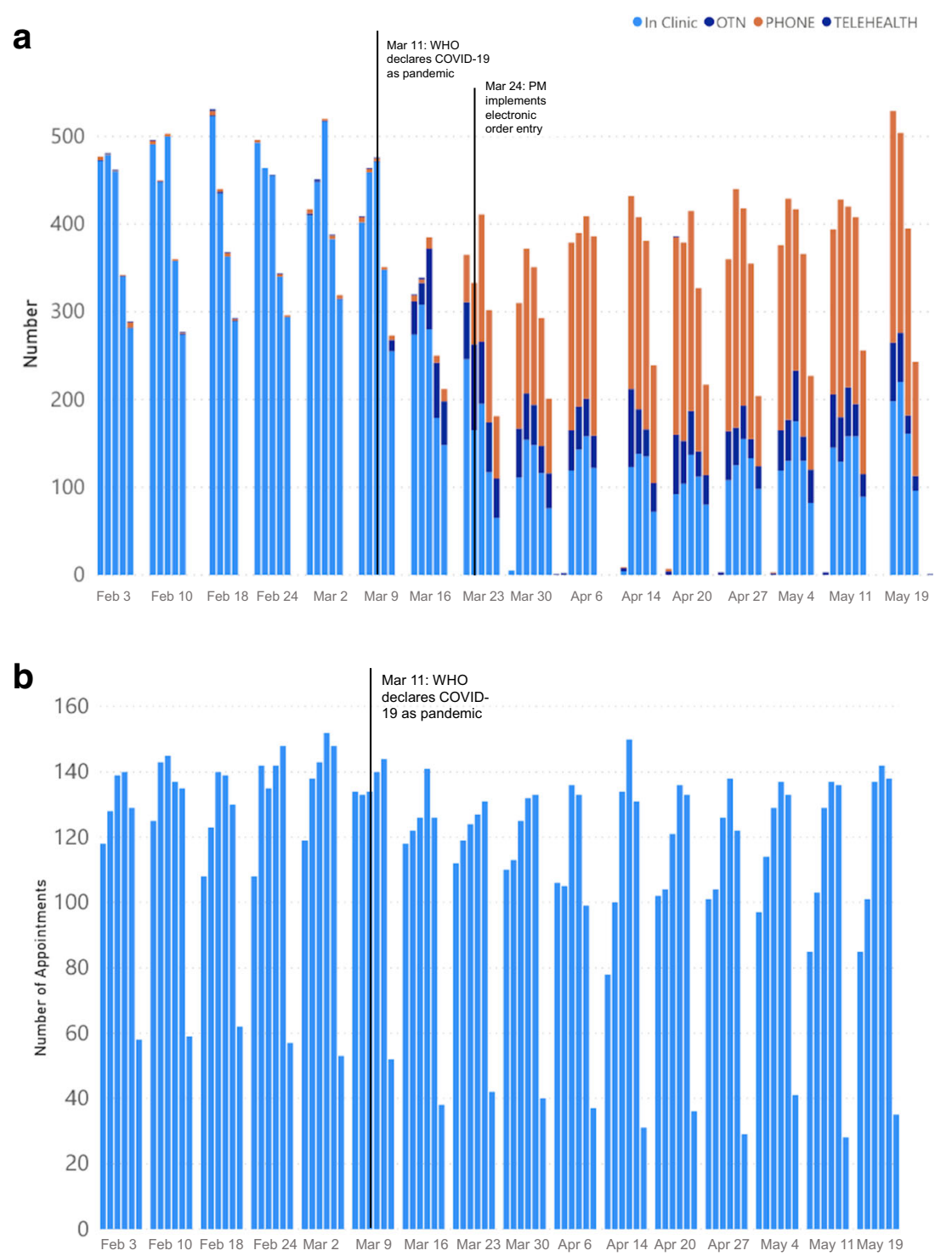

premises physical distancing, minimizing the risk of exposure for patients who must attend in-person to receive systemic therapy (Fig. 1b).

These strategies for risk mitigation have promoted new dimensions of patient-centered care. Although they have been implemented in response to COVID-19, it is almost certain that many of these adaptations will permanently transform cancer care.

\section{Looking Ahead}

For most institutions, there will likely be an influx of patients whose cancer care has been delayed during the pandemic. Some may present with more advanced disease and thus have poorer outcomes [2]. Resources should be in place anticipating these volumes to prevent further delays in care, while maintaining the ability to continue physical distancing rules.

One of the remarkable changes is the rapid adoption of virtual care within a span of 1 to 2 months across cancer centers worldwide. Virtual care has become an acceptable alternative to in-person visits for many patients and providers. It offers more flexibility and convenience for accessing care and reduced costs and travel burden for patients, especially those who are frail, less mobile, or live in remote areas. It may improve patient satisfaction by allowing patients to receive care in the comfort of their home and local community, providing a sense of normalcy. Many patients may also feel that their care is more tailored as they are given dedicated time by their health care team. Furthermore, virtual care technology enables increased collaboration between specialities through interdisciplinary virtual consultations and expanding 
partnerships with community care providers including primary care physicians. This, in turn, can enhance comprehensive, high-quality, multidisciplinary patient-centered care.

Cancer treatments should continue to be tailored for each individual patient. Many international cancer organizations including ASCO, ESMO, and NCCN have released guidelines on this topic. Patient safety should receive increasing consideration, and adoption of less intensive systemic therapy will receive an impetus. Nonsurgical alternatives such as chemoradiation may be increasingly utilized if surgical delays remain for a period of time. For patients who prefer less frequent visits, lengthening treatment intervals may be an option [5]. The pandemic has highlighted the urgent need for further research in these areas, and the importance of good communication and documentation of these complex discussions.

Early integration of palliative care translates into improved quality of life, mental health, prognostic understanding, and decreased health service utilization for patients with advanced cancer [6]. Early advanced planning discussions have been advocated for by physicians and patients, particularly given the potential of poor outcomes due to COVID-19 in cancer patients, and the need to strategize the use of hospital resources such as intensive care unit beds and mechanical ventilators during a surge. Going forward, these discussions should play an increasing role in defining patient-centered care from the time of diagnosis of an incurable disease, along with clear documentation of the patient's goals of care in the medical record.

With respect to clinical trials, many have temporarily halted or slowed down accrual globally, due to fewer resources, staffing, physical distancing rules, and pandemic planning. At PM, around $45 \%$ of trials have been temporarily suspended. Other trials have remained open on a case-by-case basis, especially if no alternative treatment options are available. The pandemic has highlighted the need for greater flexibility in research infrastructure and clinical trial study design and conduct [7]. As the pandemic evolved rapidly, research units, ethics boards, and financial departments needed to quickly prioritize and approve research proposals. These teams should continue to prioritize research which addresses areas of urgent need and/or has the potential to have immediate and significant impact on patient outcomes. When conducting clinical trials, while still preserving high standards of ethical conduct, patient safety, and validity, less burdensome, more efficient, and cost-saving alternatives should be increasingly considered. During the pandemic, study sponsors overall have been flexible. Beyond the pandemic, reducing unnecessary trial visits, allowing virtual study consents, visits, and electronic questionnaires, and enabling laboratory and imaging tests to be performed at accredited local facilities will likely enhance patient satisfaction and decrease cost. These pragmatic elements may also increase accrual of patients concerned about travel burden on trial such as elderly patients and those living in remote areas, who have been historically underrepresented in trials. Study meetings and monitoring site visits are now held virtually through innovative digital tools. Electronic data entry has enabled remote data completion to continue without compromising data integrity. These initiatives and capabilities may pave the way for enhanced research infrastructure and trial operations in the future.

Well-being and burnout among oncology health care providers can affect the quality of patient care [8]. With the advent of COVID-19, oncology providers had to cope with rapid and dramatic changes to the delivery of oncology care, including the use of personal protective equipment, balancing the risk of contagion, heavier workload, redeployment to areas outside of the usual scope of practice, difficult conversations, moral distress, and organizational justice [9]. These changes, along with personal needs such as childcare, sleep, and hydration, can cause significant emotional distress [9]. In response to the pandemic, a multidisciplinary executive sponsored COVID Staff Well-Being Collaborative was created with the aim of maintaining a healthy and functional workforce and a goal of preventing stress from becoming distress. It allowed for advocacy, communication, on-going needs assessments, and collaborative projects to enable connected social distancing, embedded team support and psychological first aid. These projects included online drop-in exercise and meditation classes, and expansion of individual resiliency programs, resources, and tools [10]. The accelerated implementation of these programs was due to an awareness of the need and a readiness to support oncology care providers. Evaluation of this Collaborative is underway, and it is expected that it has proved useful and should continue to flourish beyond the pandemic.

\section{Conclusions}

The COVID-19 pandemic has necessitated rapid changes to cancer care and delivery unlike anything we have encountered before. The pandemic has re-invigorated a longstanding interest and need to re-invent care. Virtual care, community-based care delivery models, and pragmatic clinical trials have the potential to transform the existing system into a much more accessible, collaborative, efficient, and patient-centered infrastructure. Collectively, institutions must continue to build on this momentum to innovate, revise, and integrate outdated and fragmented infrastructure, and rigorously evaluate the impact of these new care paradigms on patient outcomes, satisfaction, and staff wellness.

\section{Compliance with Ethical Standards}

Conflict of Interest $\quad$ MKK reports grants and personal fees from Research funding from and honorarium Eisai, grants from Research funding from Exelixis, personal fees from Honorarium from Bayer, outside the submitted work. Other authors declare that they have no conflict of interest. 
Human and Animal Rights and Informed Consent This article does not contain any studies with human or animal subjects performed by any of the authors.

\section{References}

Papers of particular interest, published recently, have been highlighted as:

- Of importance

- Of major importance

1. Mehta V, et al. Case fatality rate of cancer patients with COVID-19 in a New York hospital system. Cancer Discov. 2020. https://doi. org/10.1158/1078-0432.CCR-19-2706.

2. Lai AG, et al. Estimating excess mortality in people with cancer and multimorbidity in the COVID-19 emergency. medRxiv. 2020. https://doi.org/10.13140/RG.2.2.34254.82242.

3. Stukel TA, Schull MJ, Guttmann A, Alter DA, Li P, Vermeulen MJ, et al. Health impact of hospital restrictions on seriously ill hospitalized patients: lessons from the Toronto SARS outbreak. Med Care. 2008;46:991-7.

4. Ontario Health - Cancer Care Ontario. Pandemic planning clinical guideline for patients with cancer. 20 (2020). Available at: https:// www.accc-cancer.org/docs/documents/cancer-program- fundamentals/oh-cco-pandemic-planning-clinical-guideline_final_ 2020-03-10.pdf. (Accessed: 6th May 2020).

5. Lala M, Li TR, de Alwis DP, Sinha V, Mayawala K, Yamamoto N, et al. A six-weekly dosing schedule for pembrolizumab in patients with cancer based on evaluation using modelling and simulation. Eur J Cancer. 2020;131:68-75.

6. Bauman JR, Temel JS. The integration of early palliative care with oncology care: the time has come for a new tradition. J Natl Compr Cancer Netw. 2014;12:1763-71.

7. Waterhouse D, et al. Early impact of COVID-19 on the conduct of oncology clinical trials and long-term opportunities for transformation: findings from an American Society of Clinical Oncology survey. JCO Oncol Pract. 2020. https://doi.org/10.1200/OP.20.00275 OP. 20.00275 .

8. McFarland DC, Hlubocky F, Susaimanickam B, O'Hanlon R, Riba M. Addressing depression, burnout, and suicide in oncology physicians. Am Soc Clin Oncol Educ Book. 2019;39:590-598. https:// doi.org/10.1200/EDBK_239087.

9. Greenberg N, Docherty M, Gnanapragasam S, Wessely S. Managing mental health challenges faced by healthcare workers during COVID-19 pandemic. BMJ. 2020;368:m1211.

10. Nadler MB, Barry A, Murphy T, Prince R, Elliott MS. trategies to support health care providers during the COVID-19 pandemic. CMAJ. 2020;192:E522.

Publisher's Note Springer Nature remains neutral with regard to jurisdictional claims in published maps and institutional affiliations. 